\title{
A COMBINATORIAL PROBLEM OF SHIELDS AND PEARCY
}

\author{
STEPHEN H. SCHANUEL ${ }^{1}$
}

\begin{abstract}
Pearcy and Shields asked the following question. If $x_{1}, \ldots, x_{n}$ are positive real numbers, can one always delete a subset $D$ (possibly empty) such that the following two conditions are satisfied: (1) $\sum 1 / x_{i} \leqslant n$ (sum over all deleted terms), (2) $\sum x_{i}<1$ (sum over any interval of consecutive terms disjoint from $D$ )? In this note we show that this is always possible.
\end{abstract}

Allen Shields and Carl Pearcy [1] raised this problem: Let $x_{1}, \ldots, x_{n}$ be positive real numbers. Must there exist a subset $D \subset\{1,2, \ldots, n\}$ such that $\sum_{i \in D} 1 / x_{i} \leqslant n$, while for any interval $I=\{j, j+1, \ldots, j+k\} \subset\{1,2, \ldots, n\}$ disjoint from $D$, one has $\sum_{i \in I} x_{i}<1$ ?

More informally, one wishes to delete entries from the list $x_{1}, \ldots, x_{n}$ to ensure that no block of (originally) consecutive entries remaining has sum as large as 1 . If the cost of deleting $x_{i}$ is $1 / x_{i}$, then Shields and Pearcy suggest that the total cost need not exceed $n$.

To suitably load the induction, it seems necessary to prove a bit more. To each list $x_{1}, \ldots, x_{n}$ associate a cost function $C:(0,1] \rightarrow \mathbf{R}$ defined by

$$
C(t)=\min _{D} \sum_{i \in D} \frac{1}{x_{i}}
$$

where $D$ ranges over those subsets of $\{1,2, \ldots, n\}$ for which each interval $I$ disjoint from $D$ satisfies $\sum_{i \in I} x_{i}<1$ as before, and if $n \in I$ we strengthen the requirement to $\sum_{i \in I} x_{i}<t$. Thus $C(t)$ is the cost of deleting entries to ensure that no block of consecutive entries remaining has sum as large as 1 , and (if $x_{n}$ is not deleted) the last block has sum smaller than $t$. (I am here compelled to express my indebtedness to H. C. Enos, who is responsible for the introduction of the term "cost function" into pure mathematics in this connection.) $C(t)$ is a decreasing step function, and Shields and Pearcy want: $C(1) \leqslant n$.

I claim more is true: $\int_{0}^{1} C(t) d t \leqslant n$. With the usual conventions on empty sums, this is clear when $n=0$ (or the reader may adapt the inductive step to the case $n=1)$. Thus it suffices to prove that if $C(t)$ is the cost function for

Received by the editors November 17, 1973.

AMS (MOS) subject classifications (1970). Primary 05A20; Secondary 05B40.

${ }^{1}$ Partially supported by NSF Grant PO 28491-002. 
the sequence $x_{1}, \ldots, x_{n}$ and $c(t)$ that for $x_{1}, \ldots, x_{n-1}$, then $\int_{0}^{1} C(t) d t \leqslant 1$ $+\int_{0}^{1} c(t) d t$.

If $s_{n} \geqslant 1$ this is clear: $x_{n}$ must be deleted (no matter what the value of $t$ ) and thus

$$
C(t)=1 / x_{n}+c(1) \leqslant 1+c(t)
$$

(since $c(t)$ decreases with increasing $t$ ).

If $x_{n}<1$, estimate $C$ on two intervals for $t \leqslant x_{n}$,

$$
C(t)=1 / x_{n}+c(1) \leqslant 1 / x_{n}+c\left(1-\left(x_{n}-t\right)\right) ;
$$

(the equality because $x_{n}$ must be deleted, and the inequality because $c(t)$ decreased, for $t>x_{n}, C(t) \leqslant c\left(t-x_{n}\right)$ (the inequality because we only consider the possibility of not deleting $x_{n}$; one might improve by deleting it). Combining the two estimates we conclude

$$
\int_{0}^{1} C(t) d t \leqslant \int_{0}^{x_{n}} \frac{1}{x_{n}}+c\left(1-\left(x_{n}-t\right)\right) d t+\int_{x_{n}}^{1} c\left(t-x_{n}\right) d t ;
$$

and the right side reduces to

$$
1+\int_{1-x_{n}}^{1} c(t) d t+\int_{0}^{1-x_{n}} c(t) d t=1+\int_{0}^{1} c(t) d t
$$

as desired.

The proof above is as short as one could want, but its presentation in terms of integration of step functions rather conceals the essential geometric content. If one interprets the above proof geometrically, and takes account of the change of variable (amounting to a translation on reals mod 1), it is not difficult to see that the extremal sequences $x_{1}, \ldots, x_{n}$ (for which the ShieldsPearcy problem achieves the cost $n$ ) correspond to certain tilings (by $n$ rectangular tiles each of area 1 ) of a cylinder with base of circumference 1 and height $n$, the $x_{i}$ being the widths of the tiles.

\section{REFERENCES}

1. Allen Shields and Carl Pearcy, Almost commuting matrices (in preparation).

Department of Mathematics, State University of New York, Buffalo, New York 14222

Department of Mathematics, University of Michigan, Ann Arbor, Michigan 48104 